\title{
Measurement of the light component $(p+H e)$ energy spectrum with the DAMPE space mission
}

\author{
Francesca Alemanno, ${ }^{a, b},{ }^{*}$ Paolo Bernardini, ${ }^{c, d}$ Antonio De Benedittis, ${ }^{c, d}$ \\ Ivan De Mitri $^{a, b}$ and Zhaomin Wang ${ }^{a, b, \dagger}$ on behalf of the DAMPE Collaboration \\ (a complete list of authors can be found at the end of the proceedings) \\ ${ }^{a}$ Gran Sasso Science Institute (GSSI), \\ Via Iacobucci 2, I-67100 L'Aquila, Italy \\ ${ }^{b}$ Istituto Nazionale di Fisica Nucleare (INFN) - Laboratori Nazionali del Gran Sasso, \\ I-67100 Assergi, L’Aquila, Italy \\ ${ }^{c}$ Dipartimento di Matematica e Fisica E. De Giorgi, Università del Salento, \\ I-73100, Lecce, Italy \\ ${ }^{d}$ Istituto Nazionale di Fisica Nucleare (INFN) - Sezione di Lecce, \\ I-73100, Lecce, Italy \\ E-mail: francesca.alemanno@gssi.it
}

The DArk Matter Particle Explorer (DAMPE) is a space-based particle detector launched in a Sunsynchronous orbit on December $17^{\text {th }}, 2015$ from the Jiuquan Satellite Launch Center, in China. It has been taking data very smoothly for more than 5 years. Science goals of the DAMPE mission include the study of the electron-positron energy spectrum, the study of galactic cosmic-rays, gamma-ray astronomy, and indirect dark matter search. Performing precise measurements of light elements in space, the most abundant components of cosmic radiation, is necessary to address major problems in galactic cosmic ray acceleration and propagation mechanisms. Selecting a combined proton and helium sample (instead of proton or helium alone) allows larger efficiency and purity, also minimizing systematic effects in the reconstruction of the energy spectrum, due to possible cross-contaminations. The use of looser analysis cuts allows collecting larger statistics thus extending the covered energy range and providing a link between direct and indirect cosmicray measurements. The measurement of the p+He energy spectrum up to $\sim 150 \mathrm{TeV}$ will be presented, along with a discussion on the features of the spectrum and a comparison with other experimental results.

$37^{\text {th }}$ International Cosmic Ray Conference (ICRC 2021)

July 12 th - 23rd, 2021

Online - Berlin, Germany

\footnotetext{
${ }^{*}$ Presenter

${ }^{\dagger}$ Now at Shandong Institute of Advanced Technology (SDIAT), Jinan, Shandong, 250100, China.
} 


\section{Introduction}

The standard paradigm of a single power-law cosmic-ray (CR) spectrum up to the knee energy $(\sim \mathrm{PeV})$ seems to be not valid anymore, due to several results obtained with direct CR measurements. A first deviation from the single power-law, the so-called "hardening", has been observed at some hundreds of $\mathrm{GeV} / \mathrm{n}$ from several experiments [1-7], in their measurements on hadronic spectra, along with a spectral softening found in the DAMPE proton [8] and helium [9] spectra, at $\sim 15$ $\mathrm{TeV}$ and $\sim 34 \mathrm{TeV}$ respectively. More measurements on these unexpected features are crucial in order to have a better understanding of CR acceleration and propagation mechanisms in our galaxy [10]. The DAMPE experiment can give an important contribution to this scenario. DAMPE is a satellite, collecting data in a Sun-synchronous orbit, at $500 \mathrm{~km}$ altitude, for more than 5 years. Although it was mainly designed for precise measurements of electron and gamma-ray spectra, and for dark matter searches, DAMPE can measure cosmic rays up to a few hundreds of TeV with unprecedented energy resolution, thanks to its deep calorimeter [11]. Since proton and helium are the most abundant component of CR, it is useful to measure their energy spectra both separately and together. In particular, in this work, the measurement of $\mathrm{CR}$ proton $(\mathrm{H})+$ helium $(\mathrm{He})$ flux with energy up to $\sim 150 \mathrm{TeV}$ will be presented. Selecting $\mathrm{H}$ and He nuclei (light nuclei) together, instead of $\mathrm{H}$ or He alone, has advantages of almost no background and very high purity. Using looser analysis cuts allows collecting larger statistics thus extending the spectrum to higher energy, providing a link on the $\mathrm{H}+\mathrm{He}$ spectra between direct and indirect measurements. Furthermore, the combined light nuclei spectrum can be used as a cross-check for the independent $\mathrm{H}$ and $\mathrm{He}$ analyses eventually confirming the observed hardening and softening features.

\section{The DAMPE detector}

DAMPE is a high-energy particle detector, composed of several sub-detectors working together to provide a complete identification of the incoming particles and nuclei. Starting from the top, the first sub-detector is the plastic scintillator detector (PSD) composed of two interleaved planes of plastic scintillators, each one made by two layers of staggered bars, read-out by photomultiplier tubes (PMTs) on both ends. The aim of the PSD is to provide a charge measurement of incoming nuclei, thus identifying each different species from hydrogen $(Z=1)$ to iron $(Z=26)$. Another important task of the PSD is to separate electrons from gamma-rays: these two particles will have the same behavior in the calorimeter, but only electrons will leave a signal in the PSD. Moving downwards, the next sub-detector is the silicon tungsten tracker (STK), used for precise tracking thanks to six double layers of silicon microstrip detectors, and for gamma-rays pair production by mean of thin tungsten layers. After the STK, there is the BGO calorimeter (BGO), made by 14 interleaved layers of 22 BGO bars readout by PMTs on both ends, in which particles and nuclei produce showers, allowing electron-proton separation and energy measurement up to a few hundreds of $\mathrm{TeV}$ for nuclei, and $\sim 10 \mathrm{TeV}$ for electrons and positrons. Finally, at the bottom of the satellite, there is the neutron detector (NUD), which provides an additional hadron rejection. More details can be found in [11]. 


\section{Data sample}

This work is based on 60 months of data collected from the DAMPE satellite, from January 2016 (a few days after its launch) until December 2020, resulting in a total exposure time of $12.0 \times$ $10^{7} \mathrm{~s}$, after subtracting the dead time. Contributions to the dead time are DAMPE passing through the South Atlantic Anomaly (SAA) region ( 4.5\% of total time), the electronics response which is $\sim 3 \mathrm{~ms}$ for each triggered event ( $\sim 18 \%$ of total time), daily on-orbit calibration and monthly electronic-linearity calibration ( $\sim 1.8 \%$ of total time). In order to unfold the detector response and hence compute the spectrum, we produced detailed Monte Carlo (MC) simulations of the DAMPE detector and of proton and helium CR events between $10 \mathrm{GeV}$ and $500 \mathrm{TeV}$. These samples were simulated with the GEANT4 software, using the physics lists FTFP_BERT and EPOS-LHC (further details on this topic can be found in [8] and [9]).

\section{Selection of events}

DAMPE collects $\sim 5$ million events per day but only a part of these data will be useful for this analysis. The preselection of good quality events first, and of proton and helium events afterwards is performed through the following steps.

\subsection{Preselection}

The preselection is mainly based on BGO measurements. It consists of 1) selection of events with energy deposited in the BGO calorimeter larger than $20 \mathrm{GeV}$, to avoid the effect of the geomagnetic rigidity cutoff; 2) rejection of events entering the detector from the side, with the request of energy deposition in a single bar of the BGO lower than 35\% of the total particle energy; 3) rejection of events in which the maximum energy deposition is at the BGO edge; 4) the central axis of the shower is required to be at a distance lower than $280 \mathrm{~mm}$ from the BGO center, to ensure a good containment of the shower; 5) the track has to be fully contained inside the PSD; 6) exclusion of SAA.

\subsection{Track-selection and Trigger selection}

The preselected events will still consist of many tracks, among which we have to choose the best track. In particular, we require the match of the reconstructed tracks between BGO and STK, and between PSD and STK. Furthermore, events must activate the High Energy Trigger (HET) of DAMPE i.e. energy deposition in the top 4 BGO layers exceeding the threshold of $\sim 10$ MIPs in each hit BGO bar.

\subsection{Charge Selection}

All the previous cuts are important to ensure the quality of the selected events, but the final identification of proton and helium is done by measuring their charge in the PSD. First of all a correction for light attenuation and incident angle $[12,13]$ is performed on the signal in the PSD bars, which makes it proportional to $Z^{2}$ according to the Bethe-Bloch equation. Each PSD layer gives an independent charge measurement, which is combined in one mean value (PSD global energy) for this analysis. Being the energy range measured by DAMPE very large (from $\sim 20 \mathrm{GeV}$ 
to $\sim 100 \mathrm{TeV}$ ), we need to consider that the energy released in the PSD will be different depending on the primary energy. For this reason, the charge selection is performed in different bins of energy deposited in the BGO calorimeter. In Fig.1a an example of energy released in the PSD by proton (first peak) and helium nuclei (second peak), using 60 months of data, for energy released in the BGO between $1.6 \mathrm{TeV}$ and $2.5 \mathrm{TeV}$, fitted using a Landau function convoluted with a Gaussian function (LanGaus). In Fig.1b the total charge selection range for the $\mathrm{p}+\mathrm{He}$ analysis, defined by the MPV and sigma values extrapolated with the LanGaus fits.

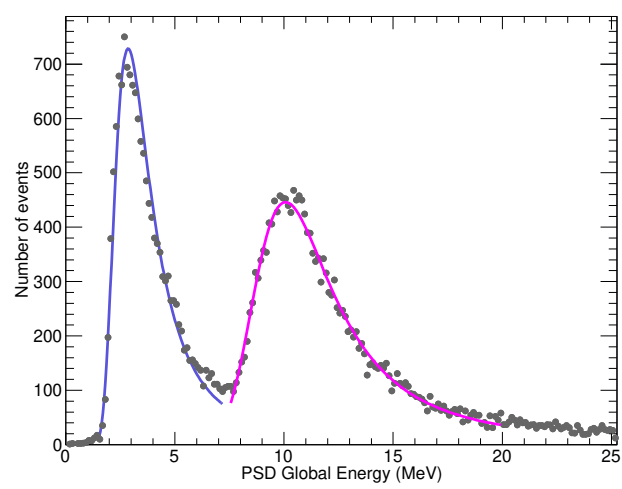

(a)

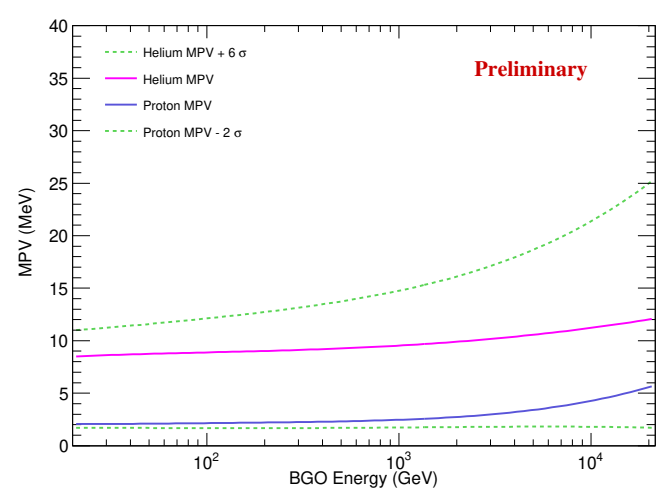

(b)

Figure 1: (a) Energy released in the PSD by proton and helium nuclei, when the BGO energy deposition is between $1.6 \mathrm{TeV}$ and $2.5 \mathrm{TeV}$. The lines are the LanGaus fit functions used for proton (blue line) and helium (magenta line). (b) Charge selection range for $\mathrm{p}+\mathrm{He}$, based on the energy released in the PSD. After fitting the charge histograms the MPV is plotted with respect to the energy released in the BGO, for both proton and helium. The continuous lines represent the polynomial functions used to fit the proton data (in blue), and the helium data (in magenta). The green dashed lines mark the limits for the charge selection: proton MPV function $-2 \sigma$ and helium MPV function $+6 \sigma$.

\section{Acceptance}

The effective acceptance of the DAMPE detector is defined as follows:

$$
\mathrm{A}_{\mathrm{acc}}^{\mathrm{i}}=\mathrm{G}_{\mathrm{gen}} \times \frac{\mathrm{N}\left(\mathrm{E}_{\mathrm{T}}^{\mathrm{i}}, \text { sel }\right)}{\mathrm{N}\left(\mathrm{E}_{\mathrm{T}}^{\mathrm{i}}\right)}
$$

with $\mathrm{G}_{\text {gen }}$ geometrical acceptance used for generating $M C$ data, $\mathrm{N}\left(\mathrm{E}_{\mathrm{T}}^{\mathrm{i}}\right)$ number of $\mathrm{MC}$ generated events in the $\mathrm{i}$-th bin of primary energy $\left(\mathrm{E}_{\mathrm{T}}\right)$, and $\mathrm{N}\left(\mathrm{E}_{\mathrm{T}}^{\mathrm{i}}\right.$, sel) number of those $\mathrm{MC}$ events surviving all selection cuts described in section 4. In Fig.2 the acceptance for various selection cuts with respect to the primary energy is presented.

\section{Unfolding procedure}

The depth of the DAMPE BGO calorimeter is of $\sim 32$ radiation lengths and $\sim 1.6$ nuclear interaction length [8]. This implies that it can contain very well electromagnetic showers, while 


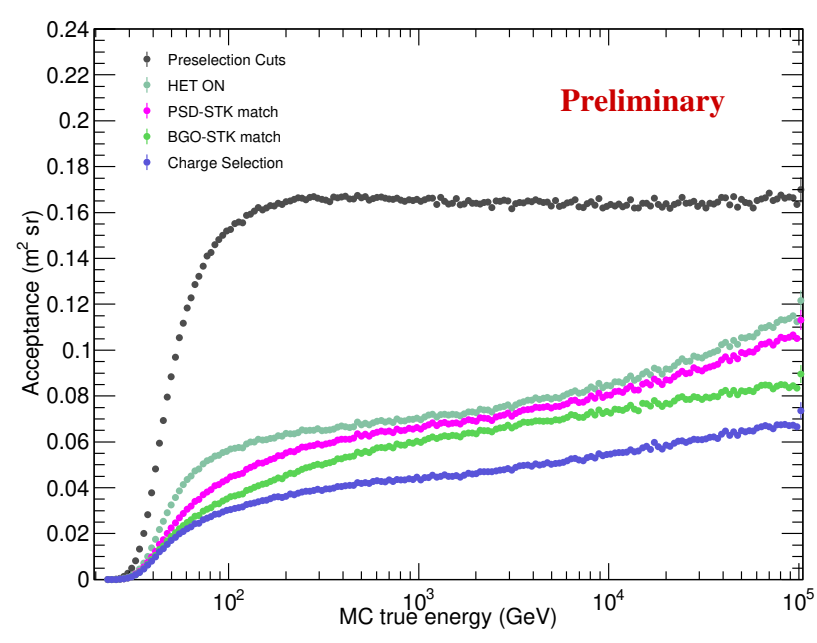

Figure 2: Effective acceptance as function of the primary energy. Each different color represents an additional cut applied on MC data, up to the charge selection which is the last one, in blue

the energy of hadronic showers will be partially lost and must be reconstructed. In particular, for $\mathrm{p}$ and He nuclei, $35 \%$ - $40 \%$ of the total energy will be deposited in the calorimeter volume. To unfold the detector response and reconstruct the primary energy, we use a method based on Bayes' theorem [14]: the detector response is estimated by using MC simulations of both hydrogen and helium nuclei; all events are required to pass the selection cuts described above; finally the unfolding method is applied, i.e. the true energy of incoming particles can be inferred from observations using the following formula:

$$
N\left(E_{T}^{i}\right)=\sum_{j=1}^{n} P\left(E_{T}^{i} \mid E_{O}^{j}\right) N\left(E_{O}^{j}\right)
$$

with $\mathrm{N}\left(\mathrm{E}_{\mathrm{T}}^{\mathrm{i}}\right)$ number of events in the $\mathrm{i}$-th bin of true energy, $\mathrm{N}\left(\mathrm{E}_{\mathrm{O}}^{\mathrm{j}}\right)$ number of observed events in the $j$-th bin of energy deposited in the calorimeter, and $P\left(E_{T}^{i} \mid E_{O}^{j}\right)$ response matrix derived from MC using the Bayes theorem (see Fig. 3).

\section{Results: proton + helium flux}

The flux for each energy bin can be written as:

$$
\Phi_{i}=\frac{N_{i}}{\Delta T \times A_{i} \times \Delta E_{i}}
$$

with $\mathrm{N}_{\mathrm{i}}$ number of events in the i-th energy bin after the unfolding, $\Delta \mathrm{T}$ total live time (see section 3), $\mathrm{A}_{\mathrm{i}}$ acceptance in the $\mathrm{i}$-th bin (see section 5), and $\Delta \mathrm{E}_{\mathrm{i}}$ representing the $\mathrm{i}$-th energy interval. In Fig. 4 the preliminary $\mathrm{p}+\mathrm{He}$ flux is presented in the energy range $50 \mathrm{GeV}-150 \mathrm{TeV}$, multiplied by $\mathrm{E}^{2.7}$, with error bars representing the statistical error, and a continuous band for the systematic uncertainties (see [15] for more details on the preliminary systematics estimation). The DAMPE proton and 


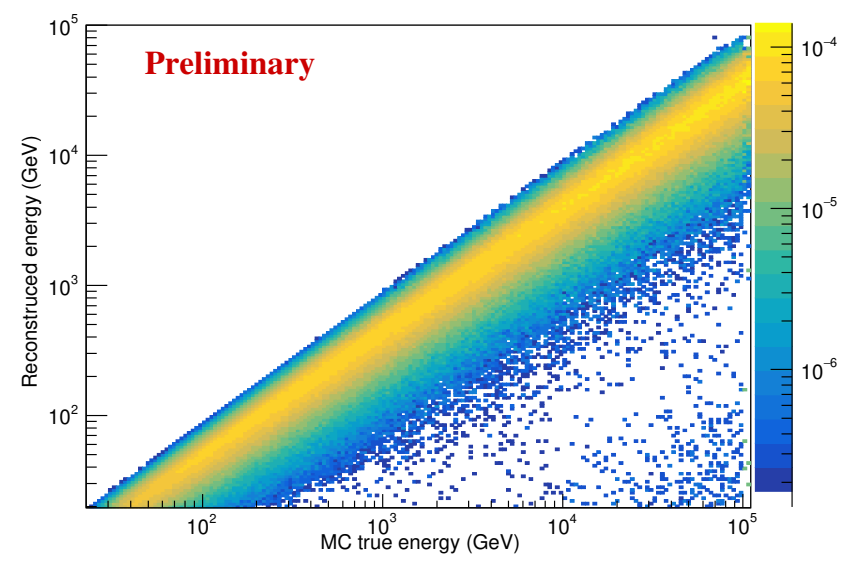

Figure 3: Response matrix derived from $\mathrm{MC}$ p and He events, passing the selection criteria

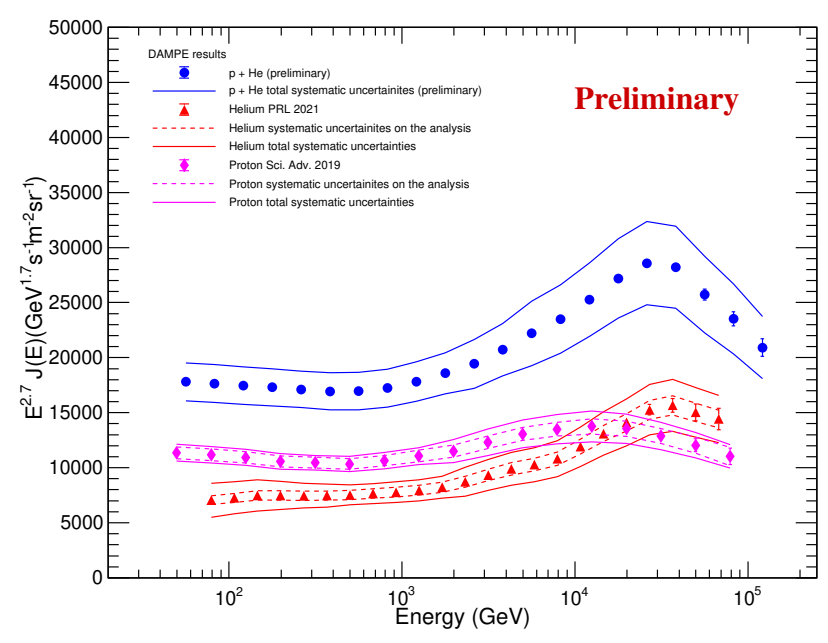

Figure 4: Preliminary p+He spectrum measured with the DAMPE detector (blue circles), between 50 $\mathrm{GeV}$ and $150 \mathrm{TeV}$. For comparison also the DAMPE proton (magenta diamonds) and helium (red triangles) spectra are shown. The error bars represent the statistical error, the dashed bands represent the systematic uncertainties from the analysis, and the continuous bands the total systematic uncertainties, including the one on the hadronic model.

helium spectra are also shown for comparison, with their respective systematic bands (for further details see [8] and [9]). The $\mathrm{p}+\mathrm{He}$ spectrum shows a spectral hardening at $\sim 600 \mathrm{GeV}$ and a softening at $\sim 25 \mathrm{TeV}$. The hardening feature confirms the result obtained by other experiments [1-7] and the DAMPE results on proton [8] and helium [9] spectra, based on independent analyses from the combined $\mathrm{p}+\mathrm{He}$ analysis shown in this work. Moreover, DAMPE reveals a softening feature in both proton and helium spectra, further confirmed by this result. The obtained values suggest rigiditydependent features, even though a mass dependence cannot be ruled out. In Fig.5, the DAMPE result is compared with direct (a) and indirect (b) p+He measurements. The DAMPE spectrum is in agreement with other direct-detection experiments, within the systematic uncertainties. The comparison with indirect measurements shows a fair general consistency, although this picture 
will be definitely clarified with the extension of the DAMPE spectrum to higher energy, which is currently in progress.

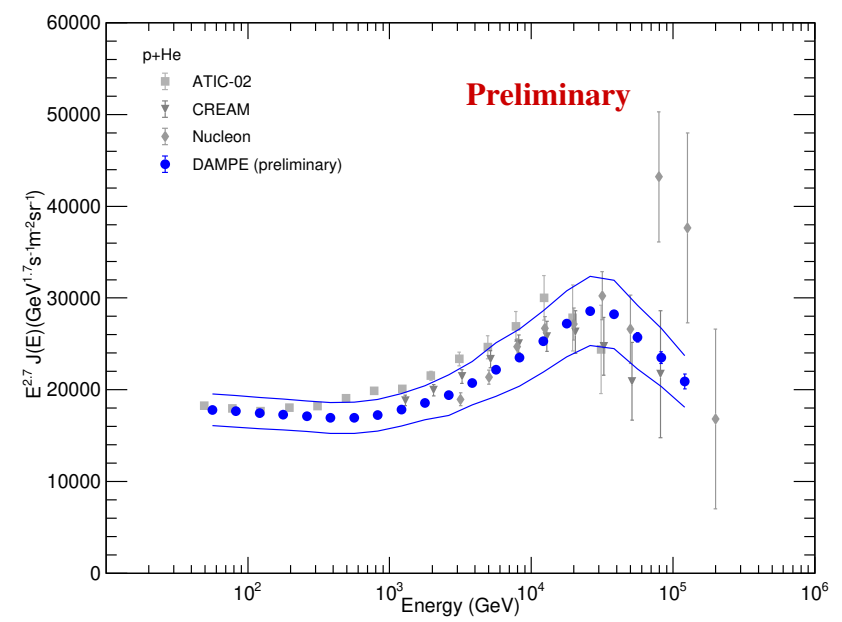

(a)

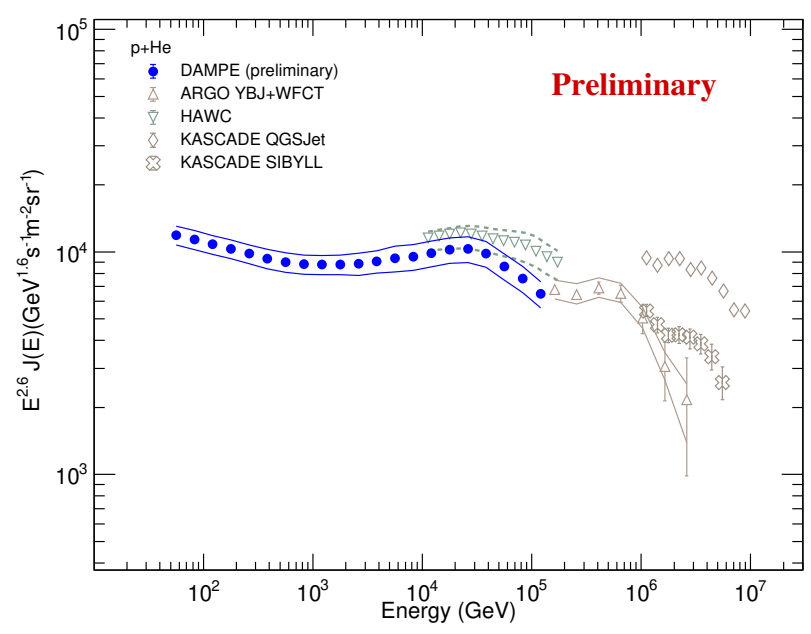

(b)

Figure 5: Preliminary p+He spectrum measured with the DAMPE detector (blue circles), between $50 \mathrm{GeV}$ and $150 \mathrm{TeV}$, compared with (a) direct measurements of p+He made by ATIC-02 [16], Nucleon [17] and CREAM [6] (b) indirect measurements from ARGO YBJ+WFCT [18], HAWC [19] and KASCADE [20].

\section{Acknowledgments}

The DAMPE mission was funded by the strategic priority science and technology projects in space science of Chinese Academy of Sciences. In China the data analysis is supported by the National Key Research and Development Program of China (No. 2016YFA0400200), the National Natural Science Foundation of China (Nos. 11921003, 11622327, 11722328, 11851305, U1738205, U1738206, U1738207, U1738208, U1738127), the strategic priority science and technology projects of Chinese Academy of 
Sciences (No. XDA15051100), the 100 Talents Program of Chinese Academy of Sciences, the Young Elite Scientists Sponsorship Program by CAST (No. YESS20160196), and the Program for Innovative Talents and Entrepreneur in Jiangsu. In Europe the activities and data analysis are supported by the Swiss National Science Foundation (SNSF), Switzerland, the National Institute for Nuclear Physics (INFN), Italy, and the European Research Council (ERC) under the European Union's Horizon 2020 research and innovation programme (No. 851103).

\section{References}

[1] O. Adriani et al. (PAMELA Collaboration), Science 332, 69 (2011); Adv. Space Res. 51 (2013), 219-226.

[2] M. Aguilar et al. (AMS Collaboration), Phys. Rev. Lett. 114, 171103 (2015); Phys. Rev. Lett. 115, 211101 (2015).

[3] M. Aguilar et al. (AMS Collaboration), Phys. Rev. Lett. 119, 251101 (2017); Phys. Rev. Lett. 120, 021101 (2018); Phys. Rev. Lett. 124, 211102 (2020); Phys. Rev. Lett. 126, 041104 (2021).

[4] O. Adriani et al. (CALET Collaboration), Phys. Rev. Lett. 122, 181102 (2019).

[5] A. D. Panov et al. (ATIC Collaboration), Bull. Russ. Acad. Sci. Phys. 73, 564 (2009).

[6] H. S. Ahn et al. (CREAM Collaboration), ApJL 714 L89 (2010); Y. S. Yoon et al. (CREAM Collaboration), ApJ 839, 5 (2017).

[7] E. Atkin et al. (NUCLEON Collaboration), JCAP07(2017)020.

[8] Q. An et al. (DAMPE Collaboration), Sci. Adv. 5, eaax3793 (2019).

[9] F. Alemanno et al. (DAMPE Collaboration), Phys. Rev. Lett. 126, 201102 (2021).

[10] Aloisio R. et al., (2018) Selected Topics in Cosmic Ray Physics, in Aloisio R. et al., (eds) Multiple Messengers and Challenges in Astroparticle Physics, Springer, Cham., and references therein.

[11] J. Chang, et al. (DAMPE Collaboration), Astropart. Phys. 95(2017) 6-24.

[12] T. Dong et al., Astropart. Phys. 105(2019) 31-36.

[13] P. X. Ma et al., Res. Astron. Astrophys. 19082 (2019).

[14] G. D’ Agostini, Nucl. Instrum. Meth.A 362 (1995) 487-498.

[15] I. De Mitri et al., PoS ICRC2019 (2021), 148.

[16] H.S.Ahn et al., Advances in Space Research, 37:1950-1954 (2006).

[17] E. Atkin et al., JCAP07(2017)020.

[18] B. Bartoli et al. (ARGO-YBJ Collaboration and LHAASO Collaboration), Phys. Rev. D, 92, 092005 (2015).

[19] J. C. Arteaga-Velázquez and J. D. Álvarez, PoS ICRC 2019, (2019) 176.

[20] K.-H. Kampert et al., Acta Phys. Pol. B, 35 (2004), p. 1799. 


\section{Full Authors List: DAMPE Collaboration}

F. Alemanno ${ }^{1,2}$, Q. An ${ }^{3,4}$, P. Azzarello ${ }^{5}$, F. C. T. Barbato ${ }^{1,2}$, P. Bernardini ${ }^{6,7}$, X. J. Bi ${ }^{8,9}$, M. S. Cai ${ }^{10,11}$, E. Casilli6, ${ }^{6,}$, E. Catanzani ${ }^{12}$, J. Chang ${ }^{10,11}$, D. Y. Chen ${ }^{10}$, J. L. Chen ${ }^{13}$, Z. F. Chen ${ }^{10,11}$, M. Y. Cui ${ }^{10}$, T. S. Cui ${ }^{14}$, Y. X. Cui ${ }^{10,11}$, H. T. Dai ${ }^{3,4}$, A. De Benedittis ${ }^{6,7}$,

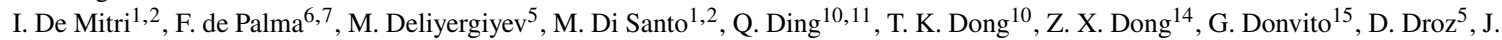
L. Duan ${ }^{13}$, K. K. Duan ${ }^{10}$, D. D’Urso ${ }^{12,1}$, R. R. Fan ${ }^{8}$, Y. Z. Fan ${ }^{10,11}$, K. Fang ${ }^{8}$, F. Fang ${ }^{13}$, C. Q. Feng ${ }^{3,4}$, L. Feng ${ }^{10}$, P. Fusco ${ }^{15,16}$, M.

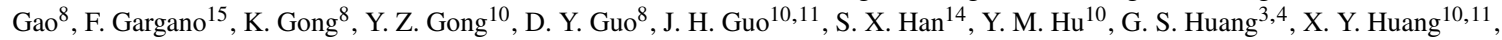

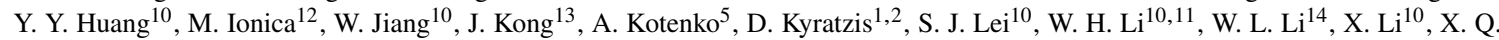

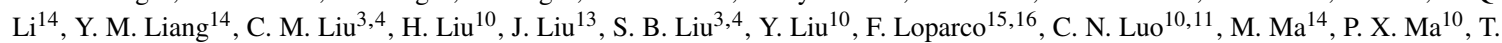

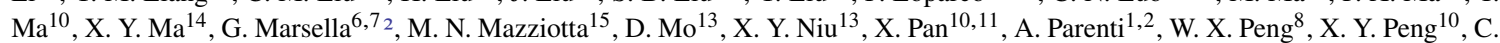
Perrina $^{5,3}$, R. Qiao ${ }^{8}$, J. N. Rao ${ }^{14}$, A. Ruina ${ }^{5}$, M. M. Salinas ${ }^{5}$, G. Z. Shang ${ }^{14}$, W. H. Shen ${ }^{14}$, Z. Q. Shen ${ }^{10}$, Z. T. Shen ${ }^{3,4}$, L. Silveri ${ }^{1,2}$, J. X. Song ${ }^{14}$, M. Stolpovskiy ${ }^{5}$, H. Su ${ }^{13}$, M. Su ${ }^{17}$, H. R. Sun ${ }^{3,4}$, Z. Y. Sun ${ }^{13}$, A. Surdo ${ }^{7}$, X. J. Teng ${ }^{14}$, A. Tykhonov ${ }^{5}$, H. Wang ${ }^{14}$, J. Z.

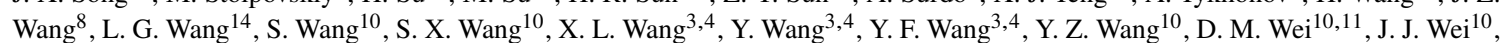
Y. F. $\mathrm{Wei}^{3,4}$, D. $\mathrm{Wu}^{8}$, J. Wu ${ }^{10,11}$, L. B. $\mathrm{Wu}^{1,2}$, S. S. $\mathrm{Wu}^{14}$, X. Wu ${ }^{5}$, Z. Q. Xia ${ }^{10}$, H. T. Xu ${ }^{14}$, Z. H. Xu ${ }^{10,11}$, Z. L. Xu ${ }^{10}$, E. H. Xu ${ }^{3,4}$, Z. Z. Xu ${ }^{3,4}$, G. F. Xue ${ }^{14}$, H. B. Yang ${ }^{13}$, P. Yang ${ }^{13}$, Y. Q. Yang ${ }^{13}$, H. J. Yao ${ }^{13}$, Y. H. Yu ${ }^{13}$, G. W. Yuan ${ }^{10,11}$, Q. Yuan ${ }^{10,11}$, C. Yue ${ }^{10}$, J. J. Zang $^{10}{ }^{4}$ S. X. Zhang ${ }^{13}$, W. Z. Zhang ${ }^{14}$, Yan Zhang ${ }^{10}$, Yi Zhang ${ }^{10,11}$, Y. J. Zhang ${ }^{13}$, Y. L. Zhang ${ }^{3,4}$, Y. P. Zhang ${ }^{13}$, Y. Q. Zhang ${ }^{10}$, Z. Zhang $^{10}$, Z. Y. Zhang ${ }^{3,4}$, C. Zhao ${ }^{3,4}$, H. Y. Zhao ${ }^{13}$, X. F. Zhao ${ }^{14}$, C. Y. Zhou ${ }^{14}$, and Y. Zhu ${ }^{14}$

${ }^{1}$ Gran Sasso Science Institute (GSSI), Via Iacobucci 2, I-67100 L'Aquila, Italy

${ }^{2}$ Istituto Nazionale di Fisica Nucleare (INFN) -Laboratori Nazionali del Gran Sasso, I-67100 Assergi, L'Aquila, Italy

${ }^{3}$ State Key Laboratory of Particle Detection and Electronics, University of Science and Technology of China, Hefei 230026, China

${ }^{4}$ Department of Modern Physics, University of Science and Technology of China, Hefei 230026, China

${ }^{5}$ Department of Nuclear and Particle Physics, University of Geneva, CH-1211, Switzerland

${ }^{6}$ Dipartimento di Matematica e Fisica E. De Giorgi, Universita‘ del Salento, I-73100, Lecce, Italy

${ }^{7}$ Istituto Nazionale di Fisica Nucleare (INFN) - Sezione di Lecce, I-73100, Lecce, Italy

${ }^{8}$ Institute of High Energy Physics, Chinese Academy of Sciences, Yuquan Road 19B, Beijing 100049, China

${ }^{9}$ University of Chinese Academy of Sciences, Yuquan Road 19A, Beijing 100049, China

${ }^{10}$ Key Laboratory of Dark Matter and Space Astronomy, Purple Mountain Observatory, Chinese Academy of Sciences, Nanjing 210023, China

${ }^{11}$ School of Astronomy and Space Science, University of Science and Technology of China, Hefei 230026, China

${ }^{12}$ Istituto Nazionale di Fisica Nucleare (INFN) - Sezione di Perugia, I-06123 Perugia, Italy

${ }^{13}$ Institute of Modern Physics, Chinese Academy of Sciences, Nanchang Road 509, Lanzhou 730000, China

${ }^{14}$ National Space Science Center, Chinese Academy of Sciences, Nanertiao 1, Zhongguancun, Haidian district, Beijing 100190, China

${ }^{15}$ Istituto Nazionale di Fisica Nucleare (INFN) - Sezione di Bari, I-70125, Bari, Italy

${ }^{16}$ Dipartimento di Fisica "M. Merlin" dell'Universita“ e del Politecnico di Bari, I-70126, Bari, Italy

${ }^{17}$ Department of Physics and Laboratory for Space Research, the University of Hong Kong, Pok Fu Lam, Hong Kong SAR, China

${ }^{1}$ Now at Dipartimento di Chimica e Farmacia, Universita“ di Sassari, I-07100, Sassari, Italy.

${ }^{2}$ Now at Dipartimento di Fisica e Chimica "E. Segre", Universita“ degli Studi di Palermo, via delle Scienze ed. 17, I-90128 Palermo, Italy.

${ }^{3}$ Also at Institute of Physics, Ecole Polytechnique Federale de Lausanne (EPFL), CH-1015 Lausanne, Switzerland.

${ }^{4}$ Also at School of Physics and Electronic Engineering, Linyi University, Linyi 276000, China. 